Cahiers de recherches médiévales

\title{
Remarques sur le motif du « service d'amour » chez quelques trouvères des cercles champenois
}

Marie-Geneviève Grossel

\section{(2) OpenEdition \\ Journals}

Édition électronique

URL : https://journals.openedition.org/crm/5893

DOI : $10.4000 / \mathrm{crm} .5893$

ISSN : 1955-2424

Éditeur

Honoré Champion

Édition imprimée

Date de publication : 20 juin 2008

Pagination : 265-276

ISSN : $1272-9752$

Référence électronique

Marie-Geneviève Grossel, «Remarques sur le motif du « service d'amour » chez quelques trouvères des cercles champenois », Cahiers de recherches médiévales [En ligne], 15 | 2008, mis en ligne le 20 juin 2011, consulté le 15 décembre 2022. URL : http://journals.openedition.org/crm/5893 ; DOI : https:// doi.org/10.4000/crm.5893 


\title{
酶M
}

\section{Remarques sur le motif du «service d'amour» chez quelques trouvères des cercles champenois ${ }^{1}$}

\begin{abstract}
Considered as one of the most topiques motives in lyric poetry of trouverres, the service d'amour, heart of the feudal metaphor, seems to have revealed all the senses( directions) which it could contain. It can however turn out interesting, in a poetic given sphere, (the champenois lyric circles), in a lapse of time confined well (roughly the first XIII ${ }^{\text {th }}$ century) to analyze how this motive was animated, particularized, even, personalized. We can give at first some elements for a semantic approach of the functioning of the motive in the lexical network where these trouveres insert it. More important, the intertextual exchanges within the social sphere, been attested or likely, allow in turn to suggest in the course of the variations, the work on a theme. We shall study finally, for the trouveres the rather important corpus of which allows to define a poetic personality, the particular treatment which they gave to this motive in their work.

Résumé : Considéré comme l'un des motifs les plus topiques dans la lyrique des trouvères, le service d'amour, cour de la métaphore féodale, semble avoir révélé tous les sens qu'il pouvait contenir. Il peut cependant s'avérer intéressant, en un milieu poétique donné, (les cercles lyriques champenois ), dans un laps de temps bien circonscrit (en gros le premier XIII siècle) d'analyser comment ce motif a été vivifié, particularisé, voire personnalisé. On peut d'abord donner quelques éléments pour une approche sémantique du fonctionnement du motif dans le réseau lexical où ces trouvères l'insèrent. Non moins importants, les échanges intertextuels au sein du milieu, attestés ou probables, permettent à leur tour de suggérer au fil des variations, le travail sur une thématique. On étudiera enfin pour les trouvères dont le corpus assez important permet de définir une personnalité poétique, le traitement particulier qu'ils ont donné à ce motif dans leur æuvre.
\end{abstract}

Dans le chapitre qu'il consacrait à l'ornement difficile, R. Dragonetti, rangeait le motif du service d'amour parmi les métaphores du vocabulaire féodal et il en décrivait le cérémonial avant d'avancer cette conclusion: "Le service de l'amant n'est qu'un art d'attendre $»^{2}$. Le simple propos de cette analyse sera de s'attacher aux mots servir/service afin d'en étudier le fonctionnement dans les chansons d'un groupe de trouvères, faciles à situer dans le temps et l'espace, celui

\footnotetext{
${ }^{1}$ Cet article reprend en l'aménageant une communication faite au Douzième Congrès de la Société Internationale de Littérature courtoise (Mythes à la cour, mythes pour la cour, Genève-Lausanne, 29 juillet-4 août 2007).

${ }^{2} \mathrm{R}$. Dragonetti, La technique poétique des trouvères dans la chanson courtoise, contribution à l'étude de la rhétorique médiévale, Bruges, de Tempel, 1960, p. 78.
}

Cahiers de Recherches Médiévales, 15, 2008 
du milieu lyrique en Champagne ${ }^{3}$. Mais rappelons brièvement le champ sémantique où évolue la notion de service.

Dès ses premiers emplois, l'idée de l'étymon servire s'est effacée pour céder la place à une soumission à la fois active et consentie ${ }^{4}$, qui apparaît tout particulièrement en contexte religieux, ainsi dans la Séquence de sainte Eulalie :

Voldrent la faire diaule servir. ${ }^{5}$

Très tôt aussi, la littérature vernaculaire emploie le verbe servir pour désigner une attitude courtoise et pleine d'urbanité réservée aux femmes, ainsi dans l'Eneas ou encore chez Chrétien : «Dames et puceles servez ${ }^{6}$ se voit recommander le jeune Perceval. C'est donc à la fois un arrière-plan de religiosité et une métaphore féodale qui vont passer dans la lyrique. On notera que le verbe servir y est beaucoup plus fréquent que son substantif service, du moins dans le corpus ici étudié.

$\mathrm{Ni}$ servir ni service ne sont des mots-rimes. Cependant certains trouvères réservent volontiers cette place d'importance à service :

Gens vilaine que je tant redoutoie

M'ont si grevé et si arriere mise

Qu'ains ne vos pou merir vostre servise ${ }^{7}$.

Comme dans ces vers de la duchesse de Lorraine, le motif n'est jamais explicité par une quelconque actualisation, à défaut de définition. Le domaine du service relève de l'implicite. La place à la rime laisse aux autres rimes qui entrent en résonance avec service le pouvoir de développer les harmoniques pour donner au mot sa tonalité propre là où le poète a choisi de l'insérer. Ces rimes sont d'ailleurs assez peu nombreuses. Parfois la rime en -ISE vient conclure la strophe en abab ab cc, les deux rimes finales acquérant de la sorte une force conclusive :

[Amour] Qui m'ocit en son servise

Qui touz sui en sa devise. ${ }^{8}$

\footnotetext{
${ }^{3}$ Pour le corpus étudié, on nous excusera de renvoyer à M-G. Grossel, Le milieu littéraire en Champagne sous les Thibaudiens, Orléans, Paradigme, 1994. Cf aussi Chansons des trouvères, chanter m'estuet, édition critique de 217 textes lyriques d'après les manuscrits, mélodies, traduction, présentation et notes de S. N. Rosenberg et H. Tischler, Paris, Lettres Gothiques, 1995.

${ }^{4}$ Voir les articles du Tobler-Lommatzsch 564-569, du Godefroy X 679 b, du FEW XI 536a.

${ }^{5}$ A. Brasseur et R. Berger, Les séquences de sainte Eulalie avec les autres poèmes du manuscrit 150 de Valenciennes, "Rithmus Teutonicus », "Dominus caeli rex ", "Uis fide », Genève, Droz, 2004. (v. 4))

${ }^{6}$ Les Romans de Chrétien de Troyes, V, Le conte du Graal (Perceval), publié par F. Lecoy,, Paris, Champion, 1975, v. 539.

${ }^{7}$ Duchesse de Lorraine, RS 1640, v. 10-12, texte dans le ms. C (BM Berne 389), édition diplomatique de J. Brakelman, Die altfranzösische Liederhandschrift Nr 389 der Stadt bibliothek zur Bern, Archiv Herrig, XLIII 1868, p. 293.
} 
On glisse ainsi de conquise/requise à mise /sanz faintise, emprise / jusqu'al juïse pour aboutir à :

Pour ce sui en son servise

Ja m'en a joie pramise. ${ }^{9}$

- ici, la force d'un serment qui engage la vie bien plus qu'une certitude d'accéder à cette joie.

Parfois les mêmes rimes amènent une assertion plus tragique encore, renforcée par l'écho d'une rime assourdie, qui se réserve la conclusion: (ligne) mise/ devise/justise...

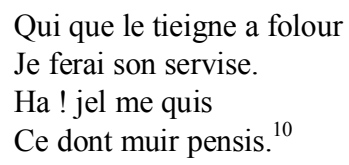

Il suffit alors d'introduire dans la ligne des mots-rimes attendus tel ou tel autre pour que la tonalité subtilement se subvertisse, ainsi chez Gautier de Dargies, fin connaisseur de l'art de Gasçoz son compainz;la ligne bien aprise/sanz faintise/justise amène avec franchise un accord en majeur, cri d'espoir et de conviction :

En li a tant de franchise

Que, se la serf, mon servise

Raverai ${ }^{11}$

tandis qu'ailleurs la ligne franchise/remise/entreprise nourrit le reprovier qui se veut discret conseil :

Je m'en plaig pour mon servise

Qui m'en est tant demourez ${ }^{12}$.

Chez Pierre de Moslins, élève appliqué et amoureux modèle, le «servise d'Amors » suit sagement la ligne guise/a devise/prise pour l'expression solennelle

${ }^{8}$ H. Petersen Dyggve, Gace Brulé, trouvère champenois, édition des chansons et étude historique, Helsinski, 1951 (Mémoires de la Soc. Néoph. De Helsingfors, XVI), XIX, RS 187, v. 7-8.

${ }^{9}$ Ibidem, v. 39-40.

${ }^{10}$ Gace Brulé, III, RS 772, v. 31-34.

${ }^{11}$ Gautier de Dargies, Poesie, edizione critica a cura di A. M. Raugei, La Nuova italia editrice, Firenze, 1981. XXIV RS 1753, v. 14-16.

${ }^{12}$ Ibidem, XIV, RS 1626, v. 7-8. 
de son sentiment ${ }^{13}$, tandis que chez Thibaut de Champagne, au gré d'un futur qui distille ses fallacieuses promesses, le service quitte le champ du pathétique pour une conquête qui n'est rien d'autre que le plaisir du chant :

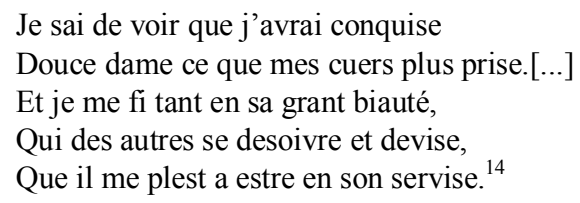

Le «service d'amour» ainsi placé à la rime pourrait se définir comme le pivot d'une proclamation de la foi, il en constitue le plege, confirmation de l'amour qui appelle la réponse de la dame, sa guise, durement déniée ou miroitant à l'horizon des attentes indéfiniment reculées.

Dans le corps du vers, «service» fonctionne en liaison avec d'autres motifs. Il est souvent enchassé dans des formules sententieuses, liées à un discours d'allure didactique :

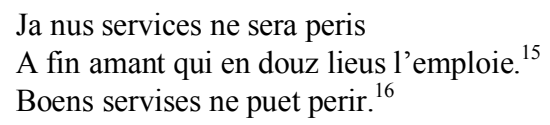

Il n'est pas davantage prétexte à une définition, que l'arrière-plan féodal soit prégnant :

On ne doit pas seigneur servise vendre

Ne vers Amors mesdire ne mesprendre. ${ }^{17}$

ou que ce soit le domaine religieux :

Et la fins est venue voirement

Que cruautez vaint merci et prïer

Ne servises n'i puet avoir mestier. ${ }^{18}$

\footnotetext{
${ }^{13}$ H.Petersen Dyygve, «Personnages historiques figurant dans la poésie lyrique des $\mathrm{XII}^{\mathrm{e}}$ et $\mathrm{XIII}^{\mathrm{e}}$ siècles, Messire Pierre de Moslins », Neuphilologische Mitteilungen, XLIII, 1942, p. $62-$ 100, I, RS 14, strophe 2.

${ }^{14}$ Les chansons de Thibaut de Champagne, roi de Navarre, édition critique publiée par A. Wallensköld, Paris, SATF, 1925. XXXVI, RS 2126, v. 23-24 et 30-32.

${ }^{15}$ Gautier de Dargies, XXII, RS 1575, v. 19-20

${ }^{16}$ Les chansons de Colin Muset, textes et mélodies éditées par Ch. Callahan et S.N. Rosenberg, V, RS 48, envoi.

${ }^{17}$ Thibaut de Champagne, IX, RS 711 v. 11-12.

${ }^{18}$ Thibaut de Champagne, XXI, RS 1479, v. 33-35.
} 
Mais, sans doute parce qu'il est lié à la notion du guerredon par l'entremise de l'expression «merir le/mon service», on peut noter que service se trouve souvent employé en des contextes de tonalité joyeuse, frémissants d'espoir :

Ne cuidiez pas, dame, ce soit folors,

Se je vos aim et serf et lo et pri,

Tant ai servi, vostre sera l'onors.

Quant vos m'avroiz mon service meri. ${ }^{19}$

Nous retrouverons cette thématique déclinée à l'infini par les anominationes de Gautier de Coinci, servir, parfait écho rimique à desservir, est glorification de la réciprocité des amours mariales. Il semble que le moine-trouvère ait été sensible à cette aura d'espérance où trouvait à se développer le motif du service enclos dans le vers, même chez Gace :
Bonement m'a asseüré
Cele $u$ mes services est saus $[\ldots]$
Je m'os bien vanter en chantant
Qu'ainc nus si loiaus n'ama,
Ne son service n'emploia
En si sage n'en si vaillant. ${ }^{20}$

Le mot service est en connexion étroite avec les règles codifiées d'un art du chant, d'un art d'aimer, cela explique peut-être qu'il puisse fonctionner comme une vérité qu'on assène et non comme l'expression déchirante de questions existentielles.

Le verbe servir recouvre des réalités plus complexes. Employé absolument, le verbe se rapproche du substantif, on trouve biau servir connoté positivement, que le contexte pathétique l'oppose en sa perfection aux cruautés du destin, comme dans ces vers de Gace :

Nus ne porroit contre sa mescheance

Si biau servir que ja li vausist nient. ${ }^{21}$

ou qu'à rebours il serve à affermir la confiance, ainsi dans la note de Colin Muset :

Je ne cuit pas ensi morir

S'ele m'i voloit retenir

En bien amer, en biau servir ;

Et du tout sui a son plesir. ${ }^{22}$

${ }^{19}$ Gace Brulé, *10, RS $1872=1884$ v. 28-31.

${ }^{20}$ Gace Brulé, *3, RS 389, v. 5-6 et 41-44.

${ }^{21}$ Gace Brulé, XXXIX, RS 233, v 21-22.

${ }^{22}$ Colin Muset, III, RS 74, v. 14-17. 
Le substantif serf, que la lyrique emploie peu, sans doute parce qu'il ressortit en d'autres registres au vocabulaire de l'insulte, est remplacé par le participe servant que nous trouvons dans une chanson de Guiot de Dijon :

Ades vait merci criant

Amors, aidiez vo servant ! $!^{23}$

et chez Gautier de Coinci :

[Notre Dame, toi)]Qui deffens et escremis

Del diable et de ses tours

Tes servanz et tes amis ${ }^{24}$.

On n'en appréciera que davantage les formules vigoureuses d'un Pierre de Moslins :
En lonc servage
Est qui aimme $[\ldots]$
Mal seignorage
Fet mal servir. $^{25}$

À l'infinitif, servir est fréquemment employé comme rime, mais développe une ligne trop diverse pour être significative. Les formules étudiées par G. Lavis ${ }^{26}$ ou R. Dragonetti, "servir en manaie/ en pardon/en esperance...», sont ici rares. Servir entre dans des couples chers à la langue médiévale, ces couples sont propres à certains trouvères, ainsi Gace affectionne "servir et honorer » là où Thibaut préfère «servir et prier», "servir et obéir»et Gautier de Coinci «servir et amer», variations intéressantes en soi qui éclairent des choix poétiques.

Le motif du service occupe la totalité du champ temporel, passé, présent, futur. Le verbe servir se trouve aussi bien dans le cadre d'une pastourelle, d'une satire, d'une chanson de croisade que dans le Grand Chant, il rejoint les emplois didactiques de service quand il est l'apanage d'un $I l$, ou d'un Qui, mais on le trouve aussi avec le $J e$ sujet. Il arrive même que le trouvère l'emploie dans un contexte péjoratif, lorsque les exigences du service d'amour poussent le fin ami à faire bonne figure à des rivaux haïs :

Esperance d'avoir alegement

Et ce qu'Amors m'a pramis a aidier

${ }^{23}$ Guiot de Dijon, Canzoni, edizione critica a cura di M. S. Lannuti, Sismel, edizioni del Galluzzo, 1999, V, RS 317, v. 6-7.

${ }^{24}$ Pour Gautier de Coinci, cf. A. Långfors, «Mélanges de poésie lyrique française », Romania LIII, p. 474-538 et Romania LVI p. 33-44. IV, RS 1836, v. 26-28.

${ }^{25}$ Ch. III RS $661=715$, v. 32-33 et 41-42.

${ }^{26} \mathrm{G}$. Lavis, L'expression de l'affectivité dans la poésie lyrique du Moyen Age (XII et XIII ${ }^{e}$ siècles), Étude sémantique et stylistique du réseau lexical « joie-dolor », Paris, Belles Lettres, 1972. 


\section{Me font chanter et rire et envoisier \\ Et honorer et servir tote gent \\ Nis les felons... ${ }^{27}$}

Pour une thématique semblable, Thibaut de Champagne remplace servir par obéir - évident synonyme ${ }^{28}$. Le verbe servir se rapproche de son étymologie quand les conditions du service le rendent à la fois incompréhensible et insupportable, c'est le motif de l'amour fatal, propre à la poétique de Gace Brulé. Plus souvent, le service dessine en filigrane la nécessaire réciproque : passant par la parole, le chant, le service ne saurait dérouler ses rites devant une dame ignorante, il en postule la présence, tout comme le fidèle ou l'officiant au service divin qui ne peut supposer vide l'espace où sa voix s'élance. La réponse- le silence éloquent, mais difficilement déchiffrable de la Dame- peut à son tour se voir marquer de réprobation, le trouvère parle alors de « servir/être servi d'ennui » :

$$
\text { De maint ennui ai puis esté servis ... }{ }^{29}
$$

C'est le propre des losengiers, dont la dame doit se garder, comme l'édicte Colin à sa belle :

Bien se doit Blondette gaitier

K'adés vuelent d'anui servir. ${ }^{30}$

Il est vrai que Colin s'autorise le jeu de mots même pour les plus graves règles d'amour lorsque chez lui le maître en l'art de trouver se mue tout soudain en maître... d'hôtel :

Ma bele douce amie $[\ldots]$

Vous serez bien servie

De crasse oës rostie ... ${ }^{31}$

Ce dernier exemple paraît particulièrement révélateur de l'aisance avec laquelle les grands trouvères se jouent de la topique, pour eux véritable espace de liberté créatrice. On peut ainsi penser que Robert de Rains, ce jongleur au talent original, n'exprime pas sans une certaine distanciation ironique le but fort précis de son service, et cela dans une chanson à l'orthodoxie courtoise impeccable :

Diex, porrai tant servir

Que nue la puisse tenir

A loisir ! ${ }^{32}$

${ }^{27}$ Guiot de Dijon, XIV, RS 647, v. 10-14.

${ }^{28}$ Ch. XVI, RS 1397, v. 10 «Por li m'estuet mainte gent obeïr ».

${ }^{29}$ Gautier de Dargies, XXII, RS 1575, v. 3.

${ }^{30}$ Colin Muset, V, v. 13-14.

${ }^{31}$ Colin Muset, III, v. 37 et 42-43. 
Robert n'est-il pas le premier, à avoir composé une sotte-chanson ?

Quintessence du chant, l'œuvre de Gace Brulé ignore le mélange des tons. Le lien qui unit l'amant à l'aimée relevant du destin, le service est ainsi justifié par sa fatalité même :

Ainz sui pour li servir nasquis
Coument que mes destraigne.

Servir s'inscrit sous le signe du toujours, il relève absolument du mode de l'intensité :

Grant poinne a et grant ire

En amor maintenir,

Plus que ne vos puis dire,

Qui tant la vuet servir. ${ }^{34}$

Si ne m'en doit repentir

Que cil qui tant servi a

Ne doit perdre por soffrir. ${ }^{35}$

Mais comme pour toute ascèse, jamais l'idéal n'est atteint :

A Guiot de Ponceaus mant

Ke nus ne puet trop servir. ${ }^{36}$

Dans la ligne des rimes qui font écho à servir entre joïr et morir, seul le second est de fait assuré, et l'alternative se résout en dilemme, ni chanter ni aimer ne sauraient connaître de terme, puisque le service se veut cheminement vers l'infini de la perfection :

Dame, mout ai petit servi

A tel don con je vos ai quis

Maiz mes cuers m'a vers vous plevi

D'estre li plus loiauz amis... ${ }^{37}$

C'est ainsi que le couple servir et honorer dévoile chez Gace la plus haute des conceptions poétiques, le servant s'y fait l'orgueilleux ministre d'une sacralisation

32 W. Mann, «Die Lieder Robert de Rains, genannt La Chievre» in Zeitschrift für rom. Philol., XXIII, 1899, p. 79-116. Ch.VI RS 1485, v. 20-23.

${ }^{33}$ Gace Brulé, I, RS 1579, couplet III « apocryphe », v 23-24.

${ }^{34}$ Ch. XVIII, RS 1757, v. 11-14.

${ }^{35}$ Ch. XX, RS 1977, v. 26-28.

${ }^{36} \mathrm{Ch}$. I, envoi.

${ }^{37}$ Ch. XIII, RS 361, v. 41-44. 
de l'art, transformant par la grâce de ses mots l'Aimée en Plus que rose, les compagnons en initiés, la chambre de la dame en thalamos, refermé sur sa mystérieuse splendeur :
Compagnon, je sai tel cose
Ki chanter me fait sovent :
Dame plus belle que rose
Ki maintient joie et jovent.
Garis est ki servir l'ose
Et qui sa merite atent
$\mathrm{K}$ 'ades est sa chambre enclose
De tous biens entierement. ${ }^{38}$

Pour Gautier de Dargies, les rituels du service d'amour se chantent sur un autre mode. Ce poète de descors excelle dans la rupture- et c'est bien ainsi que ses contemporains ont compris sa personnalité poétique.

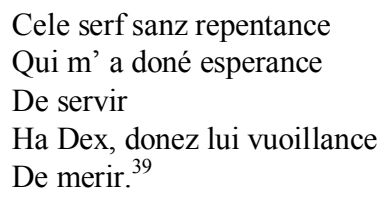

Le service sera alors une mise en scène des antithèses, mime de la balance qui marque cet amour, entre l'ire et l'espoir; non moins fier de sa valeur que Gace, Gautier fait du service la pierre de touche d'un univers manichéen où l'autre, mauvais poète mauvais amant, ne sert que de repoussoir et d'exutoire à la malédiction et ses males paroles.

Hormis Gace, un seul trouvère des cours champenoises a donné au service d'amour une place prédominante en son univers poétique, c'est Guiot de Dijon. Guiot est un trouvère de la «stricte observance", il respecte soigneusement la topique, les règles du service s'édicteront souvent sous la forme des assertions généralisantes :

\footnotetext{
Bien est Amors sans pitié

Quant ceus met en nonchaloir

Qui por li sunt envoisié

Er servent sans decevoir ${ }^{40}$
}

Sa chanson XII ${ }^{41}$ tourne ainsi autour du motif du service, fondé sur le commandement premier de la dame, service qui débuta « en enfance » par le don du

\footnotetext{
${ }^{38}$ Ch. XXIII, RS 1939, strophe I.

${ }^{39}$ Ch. XIII, v. 23-27.

${ }^{40}$ Ch. VI, RS 1088, v. 10-13.

${ }^{41} \mathrm{RS} 117$.
} 
cœur, devint ensuite un usage, une volonté, un espoir, une valeur enfin, qui englobe, avec tout l'avenir, et la mort et la vie en un précieux hysteron-proteron. Guiot, avec ce scrupuleux respect des motifs convenus, réussit à faire entendre sa tonalité propre, car la valeur de la dame jointe à la sienne finit par le laisser tout rempli de l'espoir du merir accordé à son mérite, et c'est bien suffisant pour continuer de chanter! Dans les chansons de Guiot, servir s'inscrit sous le signe du comment, fontionne avec les adverbes, bien ou plus ou lonc tans. Ici la liturgie a des aspects d'étiquette.

Comme Jocelin de Dijon, Thibaut de Champagne n'hésite nullement à parler de service d'amour en s'adressant à une bergère :

Qui set donc avoir amie

Ne servir a son talent

Fors chevalier et tel genz ? $^{42}$

Voilà qui éclaire d'un autre jour les belles déclarations dont Thibaut se veut le champion dans le Jeu-Parti :
Ne dites pas pour quoi ele vous hee
Mais servez tant et fetes-le pour quoi
Qu'ele sache ce que vostre cuers bee ;
Que par servir est maintes amors donnee. ${ }^{43}$

Le service d'amour ne se réduirait-il qu'à ce cérémonial obligé du séducteur? Il est cela aussi pour Thibaut, bien trop fin pour se faire la dupe et de la bergère aux trop vives récriminations et de soi-même, s'enchantant comme rossignol à son propre poème... Mais c'est avec une certaine indulgence que le poète s'écoute séduire, se peint courtisan docile de la trop folâtre déesse, retombe comme en quasi citation sur les mots de son maître Gace Brulé qui, pour avoir été parfait, n'en fut pas davantage eüreus :

Et se Amors estoit bien apensee,

Ele donroit a fin ami loial

Joie et secors pour sosfrir trop grant mal ;

Ensi seroit servie et honoree. ${ }^{44}$

On retrouve ici cette légère distanciation souriante qui est la personnalité poétique de Thibaut. S'y glisse aussi une subtile forme de désenchantement non point à l'égard de l'art du trouver, magnifiquement célébré durant toute une vie de roi poète, mais à l'égard du monde, des autres, et aussi peut-être de soi, quant la joie ne naît que d'éprouver et de célébrer l'amour, jamais de l'obtenir en partage :

\footnotetext{
${ }^{42}$ Pastourelle LII, RS 529, v. 22-24.

${ }^{43}$ Ch. XLIV, RS 1666, v. 11-14.

${ }^{44}$ Ch. XXX, RS 2026, v. 42-45.
} 
Mes se nus puet a bone amor venir
Par bien amer et loiament servir
Ge sai de voir qu'encor en avrai joie. ${ }^{45}$

Le service se fait ainsi liturgie de l'impossible, car qui peut se dire sûr de sa chance, maître de son cœur, juste enfin devant Dieu? De ses amours, l'on n'est jamais seigneur, sauf quand l'on n'aime plus :

Or la m'estuet servir

- Ne m'en puis plus tenir-

Et du tout obeïr

Plus qu'a rien qui soit née... ${ }^{46}$

Plutôt que fatalité, c'est une sourde inquiétude que recèle le motif, la tristesse de se savoir fragile dans un monde incertain :

Dame, se je servisse Dieu autant

Et priasse de verai cuer entier

Con je faz vous, je sai certainement

Qu'en paradis n'eüst autel loier ;

Mes je ne puis ne servir ne prier

Nului fors vos... ${ }^{47}$

Le roi-poète ne se donne pas le ridicule de se peindre en pauvre, ébloui par le parage de la dame, son emploi du motif relève du religieux, le service d'amour se mire et se confronte au service de Dieu, comme les deux faces d'un même devoir, d'une même fascination, d'une impossible réalisation :

Bien doit mes cuers estre liez et dolanz:

Dolanz de ce que je part de ma dame,

Et liez de ce que je sui desirranz

De servir Dieu qui est mes cors et m'ame. ${ }^{48}$

À sa façon qui n'était pas celle de Gautier de Dargies, Thibaut peint un service en balance, un art où l'orthodoxie parfaite n'assure pas la valeur, où la vérité de la parole n'est aucunement certitude de compréhension et de communication.

D'une cour à l'autre s'échangeaient les chansons puis d'autres trouvères aux écoutes entraient à leur tour dans le cercle magique. Lorsque Gautier de Coinci reprend le motif du service, la place extraordinaire qu'il lui concède dans ses vers montre l'importance qu'il lui voyait.

\footnotetext{
${ }^{45}$ Ch. XVI, RS 1397, v. 12-14.

${ }^{46}$ Ch. XV, RS 1268, v. 27-30.

${ }^{47}$ Ch. XXI, RS 1479, v. 21-26.

${ }^{48}$ Ch. LIV, RS 757, v. 33-36.
} 


\begin{abstract}
Douce dame qui bien te sert
L'amor ten douz fil en desert.

Bien est droiz c'on te serve.

Tuit cil qui bien te serviront

Joie sanz fin deserviront.

Diex doint je la deserve $!^{49}$
\end{abstract}

Le service de Notre-Dame, contrairement à celui du Fin Amant, est assuré d'obtenir la joie. Il n'a rien d'incertain, rien d'irréalisable, le salut est à portée d'âme, à portée d'amour, il y suffit d'aimer et de chanter Marie. Pas de balance, pas d'antithèse. Marie est aussi ferme qu'un pont jeté entre terre et ciel, aussi transparente que la fenêtre qu'elle ouvre sur l'au-delà ! Nulle demande de perfection. Le plus parfait est le simple Ave, mais rien n'empêche le trouvère de valeur de polir et ciseler ses vers chargés de feuilles et de fruits. Enfin nulle tristesse, car pour Gautier, chanter Marie et l'aimer, c'est la servir, et ce service est pures délices, véritable déduit :
A seür port touz çax mainne et conduit Qui de bon cuer entrent en sen conduit
En li servir sont tuit li grant deduit
Car c'est et fu la tres savoureuse ente
Qui touz nous paist de sen savoureus fruit. ${ }^{50}$

Comme la dame de Thibaut, plus lumineuse que le clair soleil au zénith d'un ciel estival, Marie est pure clarté; mais pour Gautier, le service de Marie possède l'avenir tout entier et sa liturgie est une liturgie de l'éblouissement.

Dans l'échange des chansons, le travail intertextuel qui cactérise l'art lyrique permet une réflexion sur les motifs essentiels chantés par les trouvères. Le mot service prend ainsi pour un temps un goût, une tonalité particulière et reconnaissable. Le motif du service d'amour renvoie non seulement à une topique générale et connue de tous, mais s'intègre dans le système que construit chaque poète de valeur auxquels ses confrères vont emprunter, avant d'y apporter les infinis miroitements de la variation. Tant il est vrai qu'une topique ne peut faire éprouver l'enchantement que si elle est une force vive.

Marie-Geneviève Grossel Université de Valenciennes et du Hainaut-Cambrésis

\footnotetext{
${ }^{49}$ Ch.I, RS 851, strophe IX.
}

${ }^{50}$ Ch. II, RS 603=748, v. 14-18. 